\title{
Patrones de resistencia a los antimicrobianos en serovares de Salmonella enterica en Perú, 2012-2015
}

\author{
Patterns of resistance to antimicrobials in serovars of Salmonella enterica in Peru, 2012-2015
}

Willi Quino', Carmen V. Hurtado', Ana María Meza', María Luz Zamudio' y Ronnie G. Gavilan

${ }^{1}$ Laboratorio de Referencia Nacional de Enteropatógenos, Centro Nacional de Salud Pública, Instituto Nacional de Salud. Lima, Perú.

Financiamiento: Instituto Nacional de Salud y CIENCIACTIVA del CONCYTEC.

Los autores declaran no tener conflictos de interés.

Recibido (segunda versión): 9 de septiembre de 2019 / Aceptado: 11 de noviembre de 2019

\section{Resumen}

Introducción: La salmonelosis es una zoonosis universal, causante de frecuentes brotes de enfermedades transmitidas por alimentos; Salmonella enterica es la especie con la mayor prevalencia, describiéndose un aumento progresivo de su resistencia a antimicrobianos. Objetivo: Determinar la frecuencia de serotipos y los patrones de resistencia antimicrobiana en aislados de $S$. enterica remitidos al Instituto Nacional de Salud, Lima, Perú. Materiales y Métodos: Se realizó un estudio descriptivo, transversal. Se incluyeron en el estudio todas las cepas remitidas como parte de la vigilancia nacional basada en laboratorio entre los años 2012 y 2015. Las cepas fueron confirmadas mediante pruebas convencionales y serotipificadas por el esquema de Kauffmann-White; la susceptibilidad antimicrobiana y la confirmación del fenotipo BLEE se realizó según el método de Kirby-Bauer y método de Jarlier. Resultados: Un total de 540 cepas de $S$. enterica fueron incluidos en el estudio, de las que $96 \%(520 / 540)$ correspondió a cepas de origen humano y 4\% (20/540) de origen no humano (aves, alimentos y ambiental). En muestras humanas, el serovar más frecuente fue $S$. Infantis (57\%), seguido de $S$. Enteritidis (27\%) y $S$. Typhimurium (6\%). Se encontró una alta resistencia a nitrofurantoína (74\%), ácido nalidíxico (64\%), ciprofloxacina (63\%), tetraciclina (63\%), ampicilina (56\%), cotrimoxazol (56\%), cefotaxima (53\%) y cloranfenicol (50\%). En muestras no humanas, el serotipo más frecuente fue $S$. Infantis (45\%), seguido de $S$. Typhimurium (40\%) y $S$. Enteritidis (10\%). encontrándose una alta resistencia a ciprofloxacina (45\%), cotrimoxazol (40\%), y tetraciclina (40\%). El 65\% del total de las cepas presentó resistencia a más de dos antimicrobianos, $43,3 \%$ fueron productoras de BLEE y $99 \%$ de éstas presentaron resistencia a entre seis y ocho antimicrobianos. Conclusiones: Se encontró una alta frecuencia de Salmonella Infantis productoras de BLEE, con multi-resistencia a los antimicrobianos en los aislados de muestras humanas y no humanas recibidas en el Instituto Nacional de Salud.

Palabras clave: Salmonella; resistencia bacteriana a antimicrobianos; $\beta$-lactamasas.

\begin{abstract}
Background: Salmonellosis is a universal zoonosis, causing frequent outbreaks of foodborne illness; Salmonella enterica is the species with the highest prevalence, a progressive increase in its resistance to antimicrobials is described. Aim: To determine the frequency of serovars and antimicrobial resistance patterns in S. enterica isolates submitted to the National Institute of Health, Lima, Peru. Methods: This is a cross-sectional study. All strains referred as part of national laboratory-based surveillance between 2012 and 2015 were included in the study. Strains were confirmed by conventional tests and serotyped by the Kauffmann-White scheme; antimicrobial susceptibility and confirmation of the BLEE phenotype was performed according to the method of Kirby-Bauer and Jarlier's method. Results: A total of 540 strains of S. enterica were included in the study, where $96 \%(520 / 540)$ corresponded to human strains and $4 \%(20 / 540)$ to non-human strains (birds, food and environmental). In human samples, the most frequent serovar was $S$. Infantis (57\%), followed by $S$. Enteritidis $(27 \%)$ and $S$. Typhimurium (6\%). High resistance to nitrofurantoin (74\%), nalidixic acid (64\%), ciprofloxacin (63\%), tetracycline (63\%), ampicillin $(56 \%)$, sulfamethoxazole-trimethoprim (56\%), cefotaxime $(53 \%)$ and chloramphenicol $(50 \%)$ was detected. In non-human samples, the most frequent serotype was $S$. Infantis (45\%), followed by $S$. Typhimurium $(40 \%)$ and $S$. Enteritidis $(10 \%)$; a high resistance to nalidixic acid (55\%), ciprofloxacin (45\%), sulfamethoxazole-trimethoprim (40\%), nitrofurantoin $(40 \%)$, tetracycline $(40 \%)$ was found. $65 \%$ of all strains had resistance to more than two antibiotics, $43,3 \%$ were ESBL producers and $99 \%$ of these had resistance between six and eight antibiotics. Conclusions: We found a high frequency of $S$. Infantis producing ESBL with multi-resistance to the antimicrobials in human and nonhuman samples received by the National Institute of Health.

Keywords: Salmonella; antibiotic resistance; $\beta$-lactamase.
\end{abstract}




\section{Introducción}

S almonella enterica es una especie bacteriana gramnegativa, facultativa intracelular, zoonótica, que constituye un problema mundial en salud pública ${ }^{1}$. La infección por Salmonella spp es una de las causas más frecuentes de las enfermedades transmitidas por alimentos (ETA) constituyendo una de las zoonosis de mayor prevalencia y uno de los principales problemas de salud pública por el incremento de su incidencia, así como la aparición de clones con fenotipos de multi-resistencia productoras de $\beta$-lactamasas de espectro extendido ${ }^{2,3}$.

Se estima que el reporte de infecciones humanas por Salmonella no tífica está por encima de 93,8 millones de casos por año, con 155.000 muertes al año a nivel mundial ${ }^{4}$. En Latinoamérica, Asia y África, la incidencia registrada de salmonelosis por año es aproximadamente de 200 a 500 casos por cada 100.000 habitantes 5 . La Autoridad Europea de Seguridad Alimentaria (EFSA) y el Centro Europeo de Prevención y Control de Enfermedades (ECDC) informan que Salmonella spp es la causa principal de ETA en Europa ${ }^{6}$. En los Estados Unidos de América (E.U.A.), Salmonella no tífica fue el segundo agente patógeno más frecuente aislado de un total de 9,4 millones de episodios de ETA y hospitalizaciones durante el período 2000-20087. En Perú, entre los años 2010 y 2012, a través de vigilancia epidemiológica, se han registrado aproximadamente 35 brotes de ETA anuales, $47 \%$ de ellos asociados a casos agudos de salmonelosis, con un total de 2.800 personas afectadas y en $51 \%$ de brotes registrados hubo entre 10 y 50 afectados 8 .

Diversos estudios han asociado el uso de antimicrobianos como promotores de crecimiento en la industria animal con el desarrollo de resistencia a antimicrobianos en bacterias patógenas responsables de causar infecciones en humanos. El uso de antimicrobianos en medicina veterinaria e industrial muestra el mismo panorama que en la práctica de la medicina humana; es decir, ejerce una presión evolutiva sobre la densidad bacteriana, además de ser factores que contribuyen a la emergencia de bacterias mejor adaptadas, y Salmonella spp no es la excepción.

La asociación entre los patógenos responsables de causar ETA en humanos y la resistencia antimicrobiana ha sido motivo de muchos estudios, principalmente en Salmonella spp., patógeno que rara vez se transfiere directamente entre personas; los alimentos serían la principal vía de contaminación en humanos?.

La salmonelosis es una de las ETA más importantes en la medicina humana y veterinaria. En muchos casos, la sintomatología es leve, y los pacientes se recuperan sin tratamiento. Sin embargo, en algunos casos, en especial niños pequeños y adultos mayores, la enfermedad puede ser grave y poner en peligro la vida, prescribiéndose algunas veces cloranfenicol, ampicilina o cotrimoxazol como alternativas terapéuticas para enfermedades diarreicas agudas de mala evolución ${ }^{10}$.

En 2010, a través de la vigilancia de laboratorio por el Instituto Nacional de Salud del Perú (INS), se detectó un incremento inusual de casos de Salmonella spp en aislados de origen humano, en su mayoría de pacientes pediátricos de diferentes hospitales de Lima, así como de alimentos. Se identificaron 33 aislados como Salmonella Infantis, 24 de origen clínico y nueve de alimentos, bacteria que estuvo asociada con el consumo de alimentos contaminados ${ }^{11}$.

La vigilancia de la salmonelosis en aislados humanos y no humanos es importante para el conocimiento de los serovares prevalentes o incidentes, para que las instituciones responsables de la salud intervengan con medidas efectivas de prevención y control de la enfermedad. Al mismo tiempo, esta vigilancia pone en evidencia el comportamiento de los serovares y la introducción de nuevos serovares en el país ${ }^{12}$.

Debido a esta realidad, el presente trabajo tuvo como objetivo determinar los serovares y los patrones de resistencia antimicrobiana en aislados de $S$. enterica remitidos al INS, Lima, Perú, durante los años 2012 a 2015.

\section{Materiales y Métodos}

Estudio descriptivo y transversal. Se incluyeron todas las cepas de $S$. enterica, aisladas por los laboratorios regionales, hospitales e instituciones privadas durante enero de 2012 a diciembre de 2015. Las cepas se recibieron en el Laboratorio de Referencia Nacional de Enteropatógenos (LRNE) del INS para la confirmación del aislado, determinación de la serovariedad y susceptibilidad antimicrobiana.

\section{Tipificación bacteriana}

Para la confirmación de los aislados de Salmonella spp., las cepas fueron pre-cultivadas en caldo tripticasa de soya (Oxoid, Inglaterra) durante 6 a 8 horas; posteriormente, cada cepa fue sembrada por dispersión y agotamiento en agar Salmonella-Shigella (Oxoid, Inglaterra) e incubada a $37^{\circ} \mathrm{C}$ por 18 a $24 \mathrm{~h}$. Luego, se procedió a la identificación mediante las pruebas bioquímicas convencionales con agar tres azucares hierro, agar lisina hierro, agar sulfuro indol movilidad y agar citrato de Simmons (Oxoid, Inglaterra). La serovariedad se desarrolló de acuerdo con el esquema de Kauffmann-White, utilizando sueros somáticos y flagelares según las indicaciones del fabricante (Denka Seiken Co., Ltd, Japón).

\section{Susceptibilidad antimicrobiana}

La susceptibilidad a los antimicrobianos se realizó a través del método de difusión por discos de Kirby-Bauer ${ }^{13}$, Los patrones de resistencia antimicrobiana se definieron 
de acuerdo a los criterios de interpretación propuestos por el Clinical \& Laboratory Standards Institute (CLSI-2015). Se probaron 13 antimicrobianos: ampicilina $(10 \mu \mathrm{g})$, amoxicilina/clavulánico $(20 / 10 \mu \mathrm{g})$, cefotaxima $(30 \mu \mathrm{g})$, ceftazidima $(30 \mu \mathrm{g})$, cloranfenicol $(30 \mu \mathrm{g})$, ciprofloxacina $(5 \mu \mathrm{g})$, tetraciclina $(30 \mu \mathrm{g})$, cotrimoxazol $(1,25 / 23,75 \mu \mathrm{g})$, meropenem $(10 \mu \mathrm{g})$, imipenem $(10 \mu \mathrm{g})$, cefoxitina $(30$ $\mu \mathrm{g})$, ácido nalidíxico $(30 \mu \mathrm{g})$ y nitrofurantoína $(300 \mu \mathrm{g})$ (Oxoid, Inglaterra). (Estos dos últimos empleados como marcadores de resistencia, aunque sin trascendencia para estas patologías en medicina humana).

Para el control de calidad se emplearon las cepas de referencia Escherichia coli ATCC 25922, Klebsiella pneumoniae ATCC 700603 y Pseudomonas aeruginosa ATCC 27853. Las cepas fueron consideradas multiresistentes cuando presentaron resistencia a más de dos antimicrobianos ${ }^{(13)}$. Adicionalmente, la determinación de la producción de $\beta$-lactamasas de espectro extendido (BLEE), se realizó mediante el método de Jarlier ${ }^{(14)}$. La presencia de BLEE se manifestó por el efecto sinérgico entre el inhibidor y los discos de antimicrobianos.

\section{Aspectos éticos}

El presente reporte se realizó como parte del protocolo "Epidemiología molecular y genética de la salmonelosis multiresistente", el que fue aprobado por el Comité de Ética del INS del Perú.

\section{Resultados}

Se analizaron 540 aislados de Salmonella enterica, 520 (96\%) de origen humano y 20 (4\%) de origen no humano. Del grupo humano, $80 \%$ correspondía a coprocultivo y $17 \%$ fue de origen extra-intestinal. Del grupo no humano, $75 \%$ se aisló a partir de aves de corral, $20 \%$ de alimentos y $5 \%$ de muestras ambientales (Tabla 1 ).

Se identificaron 16 serovares diferentes de $S$. enterica. De las muestras humanas, el serovar más frecuentemente aislado fue Salmonella Infantis (57\%), seguido de Salmonella Enteritidis (27\%) y Salmonella Typhimurium (6\%). De las muestras no humanas, el serovar más frecuente fue $S$. Infantis (45\%), seguido de $S$. Typhimurium (40\%) y $S$. Enteritidis (10\%) (Tabla 2).

La resistencia de los aislados de Salmonella spp., según el orden de su frecuencia en muestras humanas fue: nitrofurantoína (74\%), ácido nalidíxico (64\%), ciprofloxacina $(63 \%)$, tetraciclina $(63 \%)$, ampicilina $(56 \%)$, cotrimoxazol (56\%), cefotaxima (53\%) y cloranfenicol (50\%) (Tabla 3). En muestras no humanas, el orden de frecuencia fue: ácido nalidíxico $(55 \%)$, ciprofloxacina (45\%), cotrimoxazol, nitrofurantoína y tetraciclina (40\%), (Tabla 4). La mayor cantidad de perfiles distintos se encontraron dentro de $S$. Infantis, $S$. Enteritidis y $S$. Typhimurium (datos no mostrados).
Tabla 1. Distribución de serovares de Salmonellas spp. según origen de muestras. Laboratorio de Referencia Nacional de Enteropatógenos, Instituto Nacional de Salud, Perú. 2012-2015

\begin{tabular}{|c|c|c|c|c|c|c|}
\hline \multirow[t]{2}{*}{ Serotipo } & \multirow{2}{*}{$\begin{array}{c}\text { Humano } \\
\text { (H) }\end{array}$} & \multicolumn{4}{|c|}{ No humano (Nh) } & \multirow{2}{*}{$\begin{array}{c}\text { Total } \\
(\mathrm{H}+\mathrm{Nh})\end{array}$} \\
\hline & & Aves & Alimento & Ambiental & $\begin{array}{c}\text { Subtotal } \\
\mathrm{Nh}\end{array}$ & \\
\hline Salmonella Infantis & 296 & 7 & 1 & 1 & 9 & 305 \\
\hline Salmonella Enteritidis & 140 & 1 & 1 & 0 & 2 & 142 \\
\hline Salmonella Typhimurium & 29 & 6 & 2 & 0 & 8 & 37 \\
\hline Salmonella Choleraesuis & 3 & 0 & 0 & 0 & 0 & 3 \\
\hline Salmonella Blegdan & 8 & 0 & 0 & 0 & 0 & 8 \\
\hline Salmonella Paratyphi B & 5 & 0 & 0 & 0 & 0 & 5 \\
\hline Salmonella Gallinarum & 0 & 1 & 0 & 0 & 1 & 1 \\
\hline Salmonella Agona & 1 & 0 & 0 & 0 & 0 & 1 \\
\hline Salmonella Anatum & 1 & 0 & 0 & 0 & 0 & 1 \\
\hline Salmonella Corvallis & 1 & 0 & 0 & 0 & 0 & 1 \\
\hline Salmonella Derby & 3 & 0 & 0 & 0 & 0 & 3 \\
\hline Salmonella Dublin & 1 & 0 & 0 & 0 & 0 & 1 \\
\hline Salmonella Moscow & 1 & 0 & 0 & 0 & 0 & 1 \\
\hline Salmonella Reinickendorf & 1 & 0 & 0 & 0 & 0 & 1 \\
\hline Salmonella Rostock & 1 & 0 & 0 & 0 & 0 & 1 \\
\hline Salmonella spp. & 27 & 0 & 0 & 0 & 0 & 27 \\
\hline Salmonella Typhi & 2 & 0 & 0 & 0 & 0 & 2 \\
\hline Total & 520 & 15 & 4 & 1 & 20 & 540 \\
\hline
\end{tabular}

Tabla 2. Distribución anual de serovares de Salmonellas spp. en humanos (H) y no humanos (Nh) y porcentaje total de aislamiento. Laboratorio de Referencia Nacional de Enteropatógenos, Instituto Nacional de Salud, Perú. 2012-2015

\begin{tabular}{|c|c|c|c|c|c|c|c|c|c|c|c|c|}
\hline \multirow[t]{2}{*}{ Serotipo } & \multicolumn{2}{|c|}{2012} & \multicolumn{2}{|c|}{2013} & \multicolumn{2}{|c|}{2014} & \multicolumn{2}{|c|}{2015} & \multicolumn{2}{|c|}{ Total H } & \multicolumn{2}{|c|}{ Total Nh } \\
\hline & H & $\mathrm{Nh}$ & H & $\mathrm{Nh}$ & H & $\mathrm{Nh}$ & H & $\mathrm{Nh}$ & $\mathrm{n}$ & $\%$ & $\mathbf{n}$ & $\%$ \\
\hline Salmonella Infantis & 43 & 7 & 109 & 2 & 86 & 0 & 58 & 0 & 296 & 57 & 9 & 45 \\
\hline Salmonella Enteritidis & 66 & 1 & 36 & 0 & 15 & 0 & 23 & 1 & 140 & 27 & 2 & 10 \\
\hline Salmonella Typhimurium & 11 & 4 & 3 & 4 & 3 & 0 & 12 & 0 & 29 & 6 & 8 & 40 \\
\hline Salmonella Choleraesuis & 2 & 0 & 0 & 0 & 0 & 0 & 1 & 0 & 3 & 1 & 0 & 0 \\
\hline Salmonella Blegdan & 8 & 0 & 0 & 0 & 0 & 0 & 0 & 0 & 8 & 2 & 0 & 0 \\
\hline Salmonella Paratyphi B & 0 & 0 & 1 & 0 & 1 & 0 & 3 & 0 & 5 & 1 & 0 & 0 \\
\hline Salmonella Gallinarum & 0 & 1 & 0 & 0 & 0 & 0 & 0 & 0 & 0 & 0 & 1 & 5 \\
\hline Salmonella Agona & 0 & 0 & 0 & 0 & 0 & 0 & 1 & 0 & 1 & 0 & 0 & 0 \\
\hline Salmonella Anatum & 0 & 0 & 0 & 0 & 1 & 0 & 0 & 0 & 1 & 0 & 0 & 0 \\
\hline Salmonella Corvallis & 0 & 0 & 0 & 0 & 0 & 0 & 1 & 0 & 1 & 0 & 0 & 0 \\
\hline Salmonella Derby & 0 & 0 & 1 & 0 & 0 & 0 & 2 & 0 & 3 & 1 & 0 & 0 \\
\hline Salmonella Dublin & 1 & 0 & 0 & 0 & 0 & 0 & 0 & 0 & 1 & 0 & 0 & 0 \\
\hline Salmonella Moscow & 0 & 0 & 1 & 0 & 0 & 0 & 0 & 0 & 1 & 0 & 0 & 0 \\
\hline Salmonella Reinickendorf & 0 & 0 & 0 & 0 & 1 & 0 & 0 & 0 & 1 & 0 & 0 & 0 \\
\hline Salmonella Rostock & 0 & 0 & 0 & 0 & 0 & 0 & 1 & 0 & 1 & 0 & 0 & 0 \\
\hline Salmonella spp. & 0 & 0 & 26 & 0 & 1 & 0 & 0 & 0 & 27 & 5 & 0 & 0 \\
\hline Salmonella Typhi & 1 & 0 & 1 & 0 & 0 & 0 & 0 & 0 & 2 & 0 & 0 & 0 \\
\hline Total & 132 & 13 & 178 & 6 & 108 & 0 & 102 & 1 & 520 & 100 & 20 & 100 \\
\hline
\end{tabular}


Tabla 3. Resistencia antimicrobiana de las cepas de Salmonellas spp. aislados de muestras de origen humano (n: 520). Laboratorio de Referencia Nacional de Enteropatógenos, Instituto Nacional de Salud, Perú. 2012-2015

\begin{tabular}{|c|c|c|c|c|c|c|c|c|c|c|c|c|c|c|c|}
\hline Antimicrobianos & 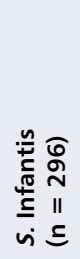 & 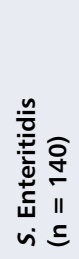 & 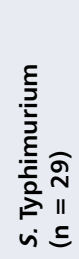 & 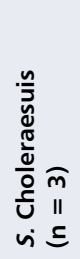 & 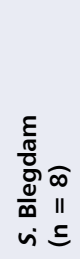 & 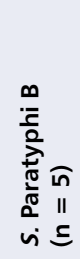 & 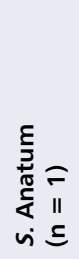 & $\begin{array}{l}\frac{.5}{0}= \\
\frac{0}{3}= \\
\text { v } \subseteq\end{array}$ & 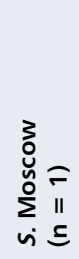 & 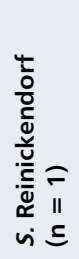 & 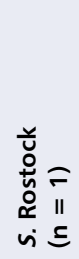 & 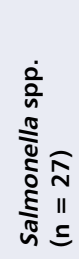 & 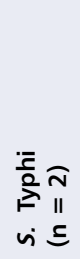 & 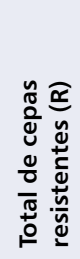 & 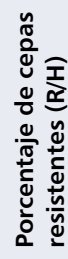 \\
\hline Ampicilina & 270 & 8 & 3 & 2 & 2 & 0 & 1 & 0 & 0 & 1 & 0 & 4 & 1 & 292 & $56 \%$ \\
\hline Cloranfenicol & 247 & 3 & 2 & 2 & 0 & 0 & 0 & 0 & 0 & 0 & 0 & 4 & 1 & 259 & $50 \%$ \\
\hline Cotrimoxazol & 274 & 5 & 2 & 3 & 0 & 0 & 0 & 0 & 0 & 1 & 0 & 4 & 1 & 290 & $56 \%$ \\
\hline Ciprofloxacina & 287 & 16 & 8 & 3 & 0 & 1 & 0 & 0 & 0 & 1 & 1 & 8 & 1 & 326 & $63 \%$ \\
\hline Ácido nalidíxico & 293 & 16 & 9 & 3 & 0 & 1 & 0 & 1 & 0 & 1 & 1 & 8 & 1 & 334 & $64 \%$ \\
\hline Nitrofurantoína & 291 & 74 & 2 & 3 & 4 & 0 & 0 & 0 & 0 & 0 & 0 & 9 & 1 & 384 & $74 \%$ \\
\hline Tetraciclina & 291 & 7 & 7 & 3 & 1 & 1 & 0 & 1 & 1 & 1 & 1 & 10 & 1 & 325 & $63 \%$ \\
\hline Ceftazidima & 32 & 2 & 0 & 0 & 0 & 0 & 0 & 0 & 0 & 0 & 0 & 0 & 0 & 34 & $7 \%$ \\
\hline Cefotaxima & 267 & 4 & 0 & 2 & 1 & 0 & 0 & 0 & 0 & 0 & 0 & 3 & 1 & 278 & $53 \%$ \\
\hline Amoxicilina/clavulanato & 4 & 1 & 0 & 0 & 2 & 0 & 0 & 0 & 0 & 0 & 0 & 0 & 0 & 7 & $1 \%$ \\
\hline Meropenem & 0 & 0 & 0 & 0 & 0 & 0 & 0 & 0 & 0 & 0 & 0 & 0 & 0 & 0 & $0 \%$ \\
\hline Imipenem & 0 & 0 & 0 & 0 & 0 & 0 & 0 & 0 & 0 & 0 & 0 & 0 & 0 & 0 & $0 \%$ \\
\hline Cefoxitina & 0 & 0 & 0 & 0 & 0 & 0 & 0 & 0 & 0 & 0 & 0 & 0 & 0 & 0 & $0 \%$ \\
\hline Fenotipo BLEE & 227 & 0 & 0 & 1 & 0 & 0 & 0 & 0 & 0 & 0 & 0 & 1 & 0 & 229 & $44 \%$ \\
\hline
\end{tabular}

Tabla 4. Resistencia antimicrobiana de las cepas de Salmonellas spp. aislados de muestras de origen no humano (n: 20). Laboratorio de Referencia Nacional de Enteropatógenos, Instituto Nacional de Salud, Perú. 2012-2015

\begin{tabular}{|c|c|c|c|c|c|c|c|c|c|c|}
\hline \multirow[t]{2}{*}{ Antimicrobianos } & \multicolumn{4}{|c|}{$\begin{array}{l}\text { Aves de corral } \\
\quad(n=15)\end{array}$} & \multicolumn{3}{|c|}{$\begin{array}{c}\text { Alimento } \\
(n=4)\end{array}$} & \multirow{2}{*}{$\begin{array}{c}\text { Ambiental } \\
(n=1) \\
S . \\
\text { Infantis } \\
(n=1)\end{array}$} & \multirow{2}{*}{$\begin{array}{l}\text { Total de } \\
\text { cepas } \\
\text { resistentes } \\
\text { (R) }\end{array}$} & \multirow{2}{*}{$\begin{array}{c}\text { Porcentaje } \\
\text { de cepas } \\
\text { resistentes } \\
\text { (R/Nh) }\end{array}$} \\
\hline & $\begin{array}{c}\text { S. } \\
\text { Infantis } \\
(n=7)\end{array}$ & $\begin{array}{c}S . \\
\text { Enteritidis } \\
(n=1)\end{array}$ & $\begin{array}{c}S . \\
\text { Typhimurium } \\
(n=6)\end{array}$ & $\begin{array}{c}S . \\
\text { Gallinarum } \\
(n=1)\end{array}$ & $\begin{array}{c}S . \\
\text { Infantis } \\
(n=1)\end{array}$ & $\begin{array}{c}S . \\
\text { Enteritidis } \\
(n=1)\end{array}$ & $\begin{array}{c}S . \\
\text { Typhimurium } \\
(n=2)\end{array}$ & & & \\
\hline Ampicilina & 3 & 0 & 0 & 0 & 0 & 0 & 0 & 1 & 4 & $20 \%$ \\
\hline Cloranfenicol & 3 & 0 & 0 & 0 & 0 & 0 & 0 & 1 & 4 & $20 \%$ \\
\hline Cotrimoxazol & 7 & 0 & 0 & 0 & 0 & 0 & 0 & 1 & 8 & $40 \%$ \\
\hline Ciprofloxacina & 7 & 0 & 0 & 1 & 0 & 0 & 0 & 1 & 9 & $45 \%$ \\
\hline Ácido nalidíxico & 7 & 0 & 0 & 1 & 1 & 1 & 0 & 1 & 11 & $55 \%$ \\
\hline Nitrofurantoína & 7 & 0 & 0 & 0 & 0 & 0 & 0 & 1 & 8 & $40 \%$ \\
\hline Tetraciclina & 7 & 0 & 0 & 0 & 0 & 0 & 0 & 1 & 8 & $40 \%$ \\
\hline Ceftazidima & 1 & 0 & 0 & 0 & 0 & 0 & 0 & 0 & 1 & $5 \%$ \\
\hline Cefotaxima & 3 & 0 & 0 & 0 & 1 & 0 & 0 & 1 & 5 & $25 \%$ \\
\hline Amoxicilina/clavulanato & 0 & 0 & 0 & 0 & 1 & 0 & 0 & 0 & 1 & $5 \%$ \\
\hline Imipenem & 0 & 0 & 0 & 0 & 0 & 0 & 0 & 0 & 0 & $0 \%$ \\
\hline Meropenem & 0 & 0 & 0 & 0 & 0 & 0 & 0 & 0 & 0 & $0 \%$ \\
\hline Cefoxitina & 0 & 0 & 0 & 0 & 0 & 0 & 0 & 0 & 0 & $0 \%$ \\
\hline Fenotipo BLEE & 3 & 0 & 0 & 0 & 1 & 0 & 0 & 1 & 5 & $25 \%$ \\
\hline
\end{tabular}


El 65\% (352) de los aislados de Salmonella spp. ( $S$. Infantis 301, $S$. Enteritidis 23, $S$. Typhimurium 9 y otros 19) presentaron resistencia a más de dos antimicrobianos. Asimismo, 43,3\% (n: 234) del total de cepas ( $S$. Infantis 99\%, S. Choleraesuis 0,43\%, y Salmonella spp. 0,43\%) fueron productoras de BLEE y $99 \%$ de ellas fueron resistentes a entre seis y ocho antimicrobianos (Figura 1).

La distribución de las cepas de Salmonella Infantis productoras de $\beta$-lactamasa, de acuerdo al sexo y grupo etario de los pacientes fue: $85 \%$ (153/180) niños; $2,2 \%$ (4/180) adolescentes, 3,3\% (6/180) jóvenes, 6,1\% (11/180) adultos y 3\% (6/180) adultos mayores. La frecuencia de Salmonella spp. productoras de $\beta$-lactamasas en el sexo femenino fue ligeramente superior al sexo masculino (Figura 2).

\section{Discusión}

El presente estudio encontró que la frecuencia de serovares de Salmonella spp. entre el 2012 y 2015, a partir de muestras humanas, fue $S$. Infantis (57\%), seguido de $S$. Enteritidis (27\%) y $S$. Typhimurium (6\%). En muestras no humanas, a partir de aves, fue $S$. Infantis (54\%), seguido de $S$. Typhimurium (31\%), $S$. Enteritidis y $S$. Gallinarum (8\%). En los hallazgos del 2012, los serovares con mayor frecuencia de Salmonella spp. fueron $S$. Enteritidis (50\%), seguida de $S$. Infantis (33\%) y $S$. Typhimurium (8\%). Sin embargo, nuestros resultados muestran que $S$. Infantis, ha ido desplazando en el tiempo a otras serovares, puesto que en el año 2010 fue el tercero serovar más frecuente (datos no mostrados), en el 2012 fue el segundo, y a partir del 2013 fue el serovar más frecuentemente aislado en muestras humanas, seguido de $S$. Enteritidis y $S$. Typhimurium; distribución que también fue observada en los años 2014 y 2015 (Tabla 2).

Los resultados encontrados en este estudio son similares a los informados en otros países, indicando cambios en la frecuencia de serovares. Muchos países coinciden en reportar que aproximadamente 50\% de los casos de enfermedad humana causados por Salmonella spp. mundialmente, son producidos por $S$. Enteritidis y $S$. Typhimurium, considerando a Salmonella Enteritidis como un problema de salud pública en todo el mundo ${ }^{15-17}$. Estos serovares han sido considerados causa importante de ETA en seres humanos ${ }^{18}$. Salmonella Enteritidis es actualmente el serovar más frecuente en África, Asia y Europa, y el segundo más frecuente en América del Norte y Oceanía. Globalmente, $S$. Enteritidis representa 43,5\% del total de aislados de Salmonella spp. en América Latina, mientras que los aislados más frecuentes a partir de alimentos son $S$. Typhimurium y $S$. Enteritidis ${ }^{19}$.

Salmonella enterica serovar Infantis ha ido emergiendo en los últimos años en todo el mundo. En E.U.A., $S$.

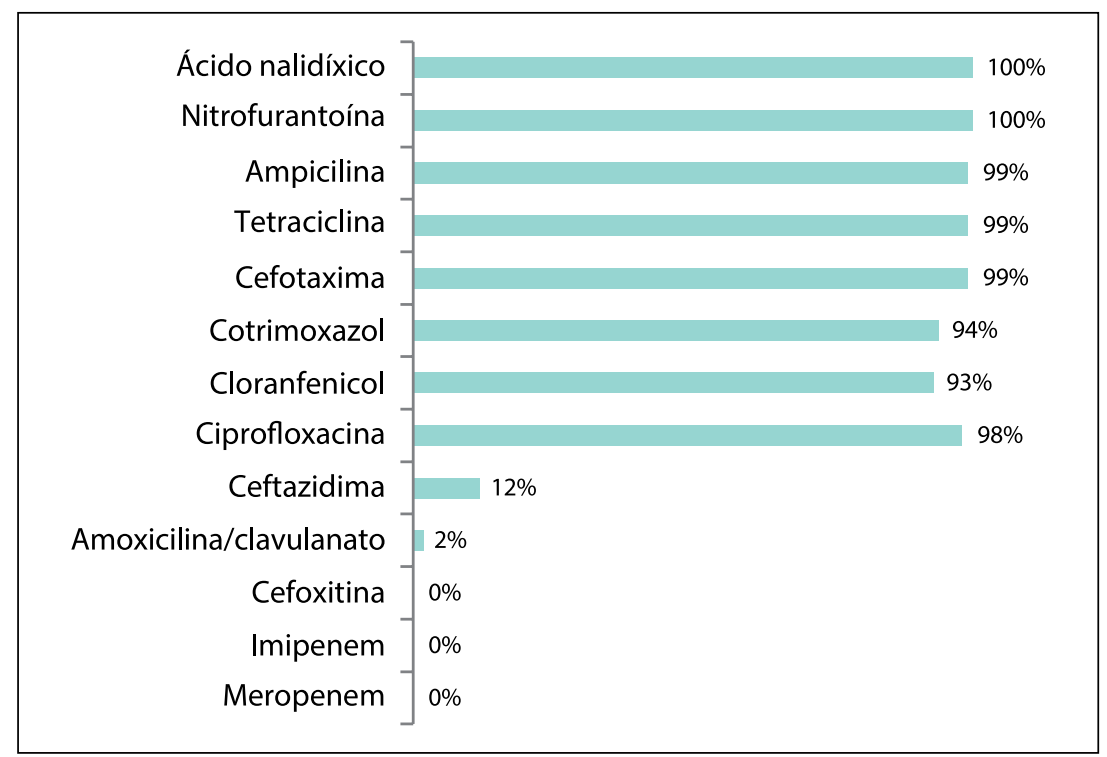

Figura 1. Porcentaje de cepas con resistencia antimicrobiana dentro del total de aislados de Salmonella con fenotipo BLEE $(n=234)$. Laboratorio de Referencia Nacional de Enteropatógenos, Instituto Nacional de Salud, Perú. 2012-2015.

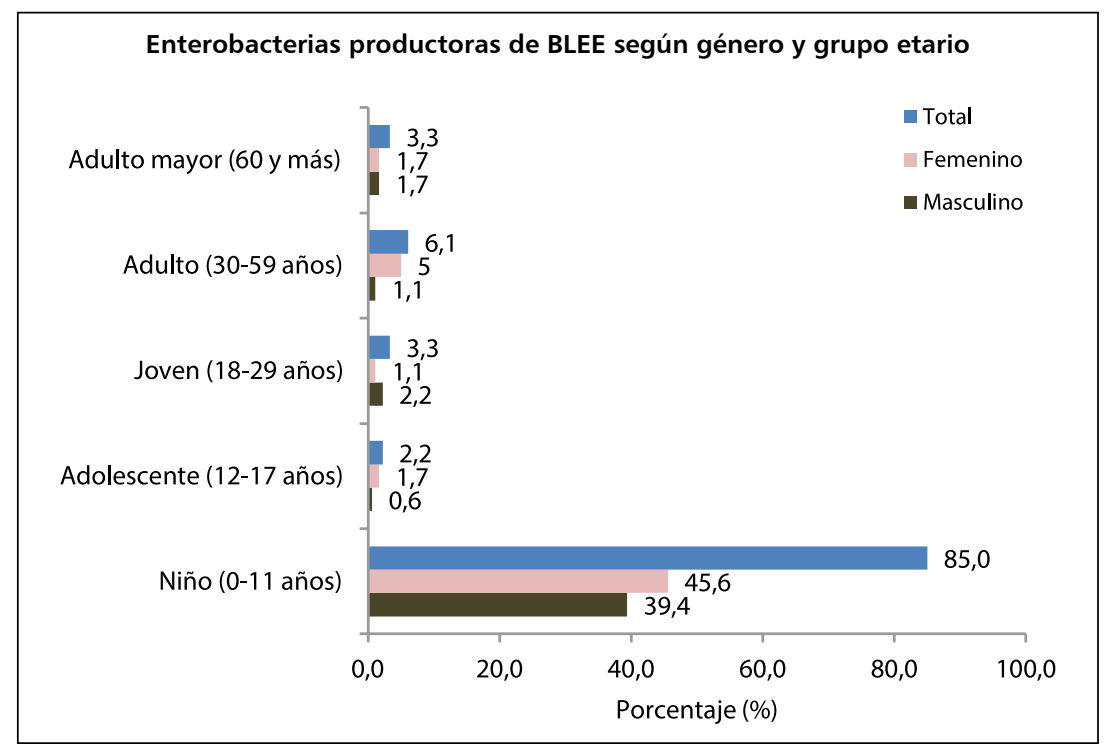

Figura 2. Frecuencia (\%) de enterobacterias productoras de BLEE de acuerdo al género y grupo etario en muestras humanas. Laboratorio de Referencia Nacional de Enteropatógenos, Instituto Nacional de Salud, Perú. 2012-2015.

Infantis ocupó el sexto lugar en el orden de prevalencia de los serovares de Salmonella ${ }^{20}$, mientras que, en la Unión Europea, fue el tercer serovar más frecuente, después de $S$. Enteritidis y Typhimurium ${ }^{21}$. En Israel, durante 2008 a 2015, S. Infantis fue el serovar predominante, responsable de $30 \%$ de todos los casos de salmonelosis en humanos ${ }^{22,23}$. 
En cuanto a la susceptibilidad antimicrobiana, tanto en aislados de muestras humanas como de muestras no humanas, se ha observado una tasa elevada de resistencia a nitrofurantoína, ácido nalidíxico, ciprofloxacina, tetraciclina, ampicilina, cotrimoxazol, cefotaxima y cloranfenicol.

En nuestro estudio, podemos considerar que la resistencia encontrada fue alta, tal como se muestra en el informe global sobre la vigilancia de la resistencia a antimicrobianos de $2014^{24}$. En muestras humanas, se observa una elevada tasa de resistencia a nitrofurantoína, tal como lo reporta la Organización Mundial de la Salud en su Informe Anual de la Red de Monitoreo/Vigilancia de la Resistencia a los Antibióticos de $2005^{25}$, lo que demuestra que el comportamiento de la resistencia bacteriana en el Perú es similar al presentado a nivel mundial, hecho preocupante ya que es un antimicrobiano ampliamente utilizado para el tratamiento y profilaxis de infecciones en diversas especies animales destinadas al consumo humano (aves, porcinos, peces) ${ }^{26}$. Esa práctica contribuye a la selección de cepas resistentes de Salmonella spp. presentes en los animales que, posteriormente, serían transmitidas al hombre.

En este estudio se evidencia que $44 \%$ de las cepas de Salmonella spp. aisladas de muestras humanas y $25 \%$ en muestras de origen no humana presentan fenotipo BLEE. En ambos casos, el serovar más frecuente con fenotipo BLEE fue $S$. Infantis, observado en 99 y $60 \%$ de los casos, respectivamente. Un estudio similar realizado en El Cairo en el año 2011, reporta una frecuencia de 63,3\% de bacterias productoras de $\mathrm{BLEE}^{27}$, mientras que otro estudio en Perú, por Colquechagua y cols., reporta una frecuencia de $64,2 \%$ de enterobacterias productoras de BLEE a partir de muestras de heces ${ }^{28}$, lo que confirma nuestros hallazgos.

La resistencia a múltiples antimicrobianos (en inglés multidrug resistance-MDR) es muy común en las bacterias productoras de $\beta$-lactamasas de espectro extendido. En este estudio se evidenció que $65 \%$ del total de cepas fueron MDR, de acuerdo a definición de MDR propuesta por Magiorakos y cols. ${ }^{29}$. En las cepas de Salmonella productoras de $\beta$-lactamasas se observa una elevada resistencia a ácido nalidíxico, nitrofurantoina, ampicilina, tetraciclina, ciprofloxacina, cotrimoxazol y cloranfenicol, siendo sensibles a meropenem, imipenem y cefoxitina. Esto es un problema grave, porque se disminuye las alternativas terapéuticas en caso de infección por estos microrganismos.

En nuestro estudio, la frecuencia de $S$. Infantis productoras de BLEE fue alta en niños bajo 11 años de edad; esto indicaría que la colonización con bacterias productoras de $\beta$-lactamasas puede ocurrir en pacientes pediátricos, lo cual puede relacionarse con el uso frecuente de antimicrobianos en la comunidad o durante las hospitalizaciones.

Entre las limitaciones de este estudio encontramos que las muestras analizadas no son necesariamente representativas de la situación nacional; sin embargo, las cepas que fueron remitidas y estudiadas en el INS constituyen una buena aproximación a lo que puede estar ocurriendo en el país. Además, ello sugiere una vigilancia epidemiológica activa tal como la que se aplica en países desarrollados. Esto implica la tipificación inmediata y sistemática de los patógenos responsables de brotes y casos, así como la fuente de origen involucrado en los mismos.

En conclusión, aunque los hallazgos de este estudio no permiten asociar las cepas aisladas de muestras no humanas con las humanas, la alta proporción de aislados que fueron resistentes a más de dos antimicrobianos, sumado a las cepas productoras de $\beta$-lactamasas resistentes a entre seis y ocho antimicrobianos, indicaría que la exposición de nuestra población a cepas de Salmonella MDR constituye un riesgo latente.

La presencia de serotipos de $S$. Infantis MDR a partir de muestras humanas en Perú evidencia la necesidad de investigar la epidemiologia molecular y genética de la salmonelosis multi-resistente.

Estudios posteriores sobre la diversidad genética, mecanismos de patogenicidad, así como la secuenciación genómica completa de las cepas de $S$. Infantis circulantes, podrían brindar información de importancia en salud pública a fin de fortalecer las políticas vinculadas a controlar la resistencia a los antimicrobianos en nuestro país.

\section{Referencias bibliográficas}

1.- Galanis E, Lo Fo Wong D M, Patrick M E, Binsztein N, Cieslik A, Chalermchikit T, et al, World Health Organization Global Salm-Surv. 2006. Web-based surveillance and global Salmonella distribution, 2000-2002. Emerg Infect Dis 12: 381-8. http://dx.doi.org/10.3201/ eid1205.050854.

2.- Sánchez M. Determinación de la prevalencia de enterobacterias del género Salmonella spp. en huevos frescos de gallina de empresas avícolas de la provincia de Tungurahua [tesis para bachiller]. Ecuador: Facultad de Medicina Veterinaria y Zootecnia. Universidad Central del Ecuador; 2013.

3.- Angulo F J, Johnson K R, Tauxe R V, Cohen M L. Origins and consequences of antimicrobial-resistant nontyphoidal Salmonella: implications for the use of fluoroquinolones in food animals. Microb Drug Resist 2000; 6 (1): 77-83. doi: 10.1089/ mdr.2000.6.77

4.- Campioni F, Moratto Bergammini A M, Falcão J P. Genetic diversity, virulence genes and antimicrobial resistance of Salmonella enteritidis isolated from food and humans over a 24-year period in Brazil. Food Microbiol 2012; 32 (2): 254-64. doi: 10.1016/j. fm.2012.06.008.

5.- Cardona-Castro N M, Sánchez-Jiménez $M$ M, Usuga-Silva L Y, Arboleda-Naranjo M, Garzón E, Velez A, et al. Characterization 
of two typhoid fever outbreaks in Apartado, Antioquia, 2005. Biomedica 2007; 27(2): 23643. doi: 10.7705/biomedica.v27i2.219.

6.- Autoridad Europea de Seguridad Alimentaria (EFSA). [Internet]. Informe de la EFSAECDC sobre intoxicaciones alimentarias en la UE 2007. Disponible en: http://www. higieneambiental.com/higiene-alimentaria/ informe-de-efsa-sobre-agentes-zoonoticos-eneuropa-2007. (Acceso el 18 de enero de 2017).

7.- Scallan E, Hoekstra R M, Angulo F J, Tauxe R V, Widdowson M A, Roy S L, et al. Foodborne illness acquired in the United States-major pathogens. Emerg Infect Dis 2011; 17(1): 7-15. doi: 10.3201/eid1701.091101p1.

8.- Boletín Epidemiológico (Perú). Enfermedades Transmitidas por Alimentos, una importante causa de morbilidad en nuestro país. Ministerio de Salud Dirección General de Epidemiología, Red Nacional de Epidemiología (Consultado el 18 de enero de 2015). Publicado en: http:// www.dge.gob.pe/portal/docs/vigilancia/ boletines/2012/50.pdf

9.- Torres Y, Zarazaga M. Repercusiones en el hombre del consumo de antibióticos por animales. Área de Bioquímica y Biología Molecular, Universidad de la Rioja, Avda. de la Paz 105, 2004 Logroño. Disponible en: http:// www.seq.es/seq/html/revista_seq/0198/rev1. html.

10.- World Health Organization. Drug-resistant Salmonella. Fact sheet N.139, Revised April 2005. Disponible en: http://www.who.int/ mediacentre/factsheets/fs139/en/index.html, (Acceso el 18 de enero de 2017).

11.- Zamudio M L, Meza A, Bailon H, MartínezUrtaza J, Campos J. Experiences in the epidemiological surveillance of foodborne pathogens by pulsed field gel electrophoresis (PFGE) in Peru. Rev Peru Med Exp Salud Publica 2011; 28 (1): 128-35.

12.- Herikstad H, Motarjemi Y, Tauxe RV. Salmonella surveillance: a global survey of public health serotyping. Epidemiol Infect 2002; 129 (1): 1-8.

13.- Bauer A W, Kirby W M, Sherris J C, Turck M. Antibiotic susceptibility testing by a standardized single disk method. Am J Clin Pathol 1966; 45 (4): 493-6. PMID: 5325707.

14.- Jarlier V, Nicolas M H, Fournier G, Philippon A. Extended broad-spectrum beta-lactamases conferring transferable resistance to newer betalactam agents in Enterobacteriaceae: hospital prevalence and susceptibility patterns. Rev Infect Dis 1988; 10 (4): 867-78. doi: 10.1093/ clinids/10.4.867.

15.- Echeita M A, Aladuena A M, Diez R, Arroyo M, Cerdan F, Gutiérrez R, et al. Serotype and phage type distribution of human Salmonella strains isolated in Spain, 1997-2001. Enferm Infecc Microbiol Clin 2005; 23 (3): 127-34. doi: $10.1157 / 13072161$.

16.- Tirado M D, Moreno R, Celades M E, Bellido-Blasco J, Pardo F J. Evolución de los serotipos, fagotipos y resistencia a antimicrobianos de Salmonella sp en el Departamento de Salud 02 de la provincial de Castellon, España (2000-2006). Rev Chilena Infectol 2009; 26 (6): 520-7. http://dx.doi. org/10.4067/S0716-10182009000700006.

17.- Pérez C, Cardozo S. Reportes de brotes y aislamientos de Salmonella spp. en Colombia. Cultura Científica, Vol 0, Iss 12, Pp 74-82 (2014). Disponible: http://www.revistasjdc. com/main/index.php/ccient/article/view/285.

18.- De Oliveira F A, Brandelli A, Tondo E C. Antimicrobial resistance in Salmonella enteritidis from foods involved in human salmonellosis outbreaks in southern Brazil. New Microbiol 2006; 29: 49-54. PMID: 16608125.

19.- Quesada A, Reginatto G, Español A, Colantonio L, Burrone M. Resistencia antimicrobiana de Salmonella spp aislada de alimentos de origen animal para consumo humano. Rev Peru Med Exp Salud Publica. 2016; 33 (1): 32-4. http://dx.doi.org/10.17843/ rpmesp.2016.331.1899.

20.- Crim S M, Griffin P M, Tauxe R, Marder E P, Gilliss D, Conquist A B, et al, Centers for Disease Control and Prevention. 2015. Preliminary incidence and trends of infection with pathogens transmitted commonly through food-foodborne diseases active surveillance network, 10 U.S. sites, 2006-2014. MMWR Morb Mortal Wkly Rep 64: 495-9.

21.- ECDC. Annual Epidemiological Report on Communicable Diseases in Europe 2009. European Centre for Disease Prevention and Control, Stockholm, Sweden. Disponible en: https://ecdc.europa.eu/sites/portal/files/ media/en/publications/Publications/0910 SUR_Annual_Epidemiological_Report_on_
Communicable Diseases_in_Europe.pdf. 22.- Aviv G, Tsyba K, Steck N, Salmon-Divon M, Cornelius A, Rahav G, et al. A unique megaplasmid contributes to stress tolerance and pathogenicity of an emergent Salmonella enterica serovar Infantis strain. Environ Microbiol 2014; 16: 977-94. http://dx.doi. org/10.1111/ 1462-2920.12351. 8 .

23.- Gal-Mor O, Valinsky L, Weinberger M, Guy S, Jaffe J, Schorr Y I, et al. Multidrug-resistant Salmonella enterica serovar Infantis, Israel. Emerg Infect Dis 2010; 16 (11): 1754-7. doi: 10.3201/eid1611.100100.

24.- Organización Panamericana de la Salud. Informe Anual de la Red de Monitoreo/ Vigilancia de la Resistencia a los Antibióticos y de Infecciones Asociadas a la Atención de la Salud-2014. Disponible en: https://www.paho. org/hq/dmdocuments/2017/2014-cha-informeanual-relavra.pdf

25.- Organización Panamericana de la Salud. Informe Anual de la Red de Monitoreo/ Vigilancia de la Resistencia a los Antibióticos, 2005. Disponible en: http://antimicrobianos. com.ar/ATB/wp-content/uploads/2013/01/ AMR_2005_Informe_Final.pdf

26.- Jones L M, Booth $\mathrm{N} \mathrm{H}$, Mcdonald L E. Farmacologia e terapeuctica em veternaría. 4. Ed. Rio de Janeiro: Guanabara Koogan;1983. p. 696-8.

27.- Abdul Rahman E M, El-Sherif R H. High rates of intestinal colonization with beta-lactamase-producing Enterobacteriaceae spread spectrum in healthy individuals. J Invest Med 2011; 59: 1284-6. doi: 10.2130/ JIM.0b013e318238748e.

28.- Colquechagua Aliaga F, Sevilla Andrad C, Gonzales Escalante E. Enterobacterias productoras de betalactamasas de espectro extendido en muestras fecales en el Instituto Nacional de Salud del Niño, Perú. Rev Peru Med Exp Salud Pública 2015; 32 (1): 26-32. http://www.scielo.org.pe/pdf/rins/v32n1/ a05v32n1.pdf.

29.- Magiorakos A P, Srinivasan A, Carey R B, Carmeli Y, Falagas M E, Giske C G, et al. Multidrug-resistant, extensively drug-resistant and pandrug-resistant bacteria: an international expert proposal for interim standard definitions for acquired resistance. Clin Microbiol Infect 2012; 18 (3): 268-81. doi: 10.1111/j.14690691.2011.03570.x 